\title{
Association of promoter methylation and 32-bp deletion of the PTEN gene with susceptibility to metabolic syndrome
}

\author{
MOHAMMAD HASHEMI ${ }^{1,2}$, HAMZEH REZAEI ${ }^{2}$, EBRAHIM ESKANDARI-NASAB ${ }^{2}$ \\ MAHMOUD-ALI KAYKHAEI $^{3}$ and MOHSEN TAHERI ${ }^{4}$ \\ ${ }^{1}$ Cellular and Molecular Research Center; Departments of ${ }^{2}$ Clinical Biochemistry and \\ ${ }^{3}$ Internal Medicine, School of Medicine; ${ }^{4}$ Genetics of Non Communicable Disease \\ Research Center, Zahedan University of Medical Sciences, Zahedan, Iran
}

Received July 1, 2012; Accepted October 30, 2012

DOI: $10.3892 / \mathrm{mmr} .2012 .1174$

\begin{abstract}
Metabolic syndrome (MeS), a cluster of several metabolic disorders, is increasingly being recognized as a risk factor for type II diabetes (T2D) and cardiovascular disease. Genetic and epigenetic alteration of the phosphatase and tensin homolog deleted on chromosome ten (PTEN) has been associated with components of MeS. The aim of the present study was to investigate the possible association of a 32-bp deletion polymorphism and promoter methylation of the PTEN gene with MeS. DNA was extracted from the peripheral blood of 151 subjects with and 149 subjects without MeS. The 32-bp deletion variant of PTEN was detected by polymerase chain reaction (PCR) and PTEN promoter methylation was defined by a nested methylation-specific PCR (MSP) method. No significant differences were found in the allelic and genotypic frequencies of the 32-bp deletion variant of PTEN between the groups [odds ratio (OR), 0.77; 95\% confidence interval (CI), 0.41-1.45; $\mathrm{P}=0.431]$. However, patients with $\mathrm{MeS}$ were identified to have lower levels of PTEN promoter hypermethylation than subjects without MeS. Promoter methylation may be a protective factor against susceptibility to $\mathrm{MeS}$ (OR, 0.52; 95\% CI, 0.29-0.92; $\mathrm{P}=0.029$ ). Our findings suggest that $P T E N$ promoter methylation may be a mechanism for PTEN downregulation or silencing in MeS, which remains to be fully clarified.
\end{abstract}

\section{Introduction}

Metabolic syndrome (MeS) is described as a combination of clinical disorders that increases the risk of obesity (central adiposity), insulin resistance, glucose intolerance, dyslipidemia, non-alcoholic fatty liver disease and cardiovascular

Correspondence to: Professor Mohammad Hashemi, Department of Clinical Biochemistry, School of Medicine, Zahedan University of Medical Sciences, Khalij Fars Blvd, Sistan and Baluchistan, Zahedan 98167-43463, Iran

E-mail:mhd.hashemi@gmail.com; hashemim@zdmu.ac.ir

Key words: metabolic syndrome, PTEN, promoter methylation, polymorphism diseases, including atherosclerosis, stroke and hypertension $(1,2)$. During the past decades, the prevalence of MeS has markedly increased worldwide, and is becoming a significant health problem (3). The etiology of this syndrome is complex and is considered to be the result of interaction between genetic and environmental factors.

Phosphatase and tensin homolog deleted on chromosome ten $(P T E N)$ was the first phosphatase to be characterized as a tumor suppressor (4). PTEN is mapped on chromosome 10q23.3 and encodes a dual lipid/protein phosphatase that acts as a negative regulator of the phosphoinositol-3-kinase (PI3K)/AKT pathway $(5,6)$. A number of studies have indicated that PTEN deficiency causes an accumulation of phosphatidylinositol 3,4,5-trisphosphate (PIP3), a lipid product of PI3K, which in turn allows constitutive activation of the PI3K/AKT signaling pathway $(7,8)$. In muscle and adipocytes, $\mathrm{PI} 3 \mathrm{~K} / \mathrm{Akt}$ pathway activation is critical for insulin-induced metabolic actions, including GLUT4 translocation, glucose uptake and glycogen synthesis (9), whereas in the liver, the PI3K/Akt pathway mediates suppression of glycogenolysis and glucose release (10). Furthermore, it has been demonstrated that PI3K/PTEN signaling pathways are significant in a variety of abnormalities associated with $\mathrm{MeS}$, including diabetes (11), obesity $(12,13)$, hypertension and cardiovascular diseases $(13,14)$. Also, it has been shown that the depletion of PTEN in the liver and adipocytes by the systemic administration of PTEN antisense oligonucleotide improved blood glucose concentrations and insulin sensitivity in diabetic mice and protected them from developing diabetes (15). However, through its protein tyrosine phosphatase activity, PTEN is able to inhibit integrin/FAK and Ras/MAPK/Erk1/2 signaling pathways under certain circumstances, hence negatively regulating cell cycling, proliferation, focal adhesion and cell migration (16). PTEN deficiency has been identified in a variety of human cancers, including glioblastoma, melanoma and cancers of the prostate, breast, lung and bladder as a result of germline or somatic mutations (17). PTEN harbors a 32-bp deletion variant located on intron 2 , but its role in disease remains unclear. Promoter hypermethylation of PTEN is considered to be an alternative to mutations and deletions as a mechanism of gene inactivation (18). Therefore, in the current study, we aimed to evaluate the association of PTEN 32-bp 
deletion polymorphism and PTEN promoter methylation with $\mathrm{MeS}$ in a sample of an Iranian population.

\section{Materials and methods}

Patients. This case control study involved 151 patients with and 149 without MeS. MeS was defined using the National Cholesterol Education Program Adult Treatment Panel III (NCEP ATP III) criteria (19) as previously described $(20,21)$. Ethical approvals for recruitment were obtained from the local ethics committee of the Zahedan University of Medical Sciences and informed consent was obtained from all individuals. The data included weight, height, waist circumference, systolic and diastolic blood pressure; blood levels of glucose, triglycerides, total cholesterol, HDL cholesterol and LDL cholesterol were collected as previously described (20-22). Blood samples were collected in EDTA-containing tubes and genomic DNA was extracted using the salting-out method as previously described (23).

The PTEN 32-bp deletion was detected using a pair of forward and reverse primers (5'-CCAGCCCTCACTAAAA ACAAA-3' and 5'-CAAGTGTCCAAGCAGCAAA-3', respectively). Polymerase chain reaction (PCR) was performed using a commercially available PCR premix (AccuPower PCR PreMix; Bioneer Corp., Daejeon, Korea) according to the manufacturer's instructions. Briefly, $1 \mu 1$ template DNA ( $\sim 100 \mathrm{ng} / \mathrm{ml}), 1 \mu \mathrm{l}$ each primer $(10 \mathrm{pmol} / \mathrm{ml})$, and $17 \mu 1$ DNasefree water were added to AccuPower PCR PreMix. Amplification was performed with an initial denaturation step at $95^{\circ} \mathrm{C}$ for $5 \mathrm{~min}$, followed by 30 cycles at $95^{\circ} \mathrm{C}$ for $30 \mathrm{sec}$, $60^{\circ} \mathrm{C}$ for $30 \mathrm{sec}$ and $72^{\circ} \mathrm{C}$ for $23 \mathrm{sec}$ with a final extension at $72^{\circ} \mathrm{C}$ for $10 \mathrm{~min}$. Each reaction was verified on a $2 \%$ agarose gel. The PCR products for insertion and deletion alleles comprised 241 and 209 bp, respectively (Fig. 1). Random samples were regenotyped to verify the accuracy of the genotyping. We identified no genotyping mistakes.

PTEN promoter methylation. The samples of DNA were treated with sodium bisulfite, which converts unmethylated $\mathrm{C}$ to $\mathrm{U}$ while methylated $\mathrm{C}$ residues resist the treatment. The method of Tiwari et al (24) was used for the bisulfite treatment of DNA with certain modifications. Briefly, $\mathrm{NaOH}$ solution was added to $10 \mu \mathrm{l}$ DNA $(\sim 1 \mu \mathrm{g})$ to a final concentration of $0.3 \mathrm{M}$. The mix was incubated at $50^{\circ} \mathrm{C}$ for $15 \mathrm{~min}$ to achieve effective denaturation of the DNA strands. This mixture was then mixed with $50 \mu 12 \%$ low melting point (LMP) agarose and incubated at $50^{\circ} \mathrm{C}$ for $15 \mathrm{~min}$. A $15-\mu 1$ drop of this mixture was pipetted into $300 \mu \mathrm{l}$ cold mineral oil (Sigma, St. Louis, MO, USA). The agarose/DNA drop solidified in the oil and agarose beads formed during incubation at $-4^{\circ} \mathrm{C}$ for $30 \mathrm{~min}$. Aliquots comprising $700 \mu \mathrm{l} 5 \mathrm{M}$ bisulfite reagent $(5 \mathrm{M}$ sodium bisulfite and $125 \mathrm{mM}$ hydroquinone; both Merck KGaA, Darmstadt, Germany; pH 5.0) were added to each reaction tube containing a single bead. The tube was gently inverted to move the bead into the aqueous phase and was incubated at $55^{\circ} \mathrm{C}$ in a water bath for $16 \mathrm{~h}$ under exclusion of light. Treatments were stopped by equilibration against $1 \mathrm{ml}$ 1X TE (2x15 min) followed by desulfonation in $500 \mathrm{ml}$ $0.2 \mathrm{M} \mathrm{NaOH}(2 \times 10 \mathrm{~min})$. Finally, the beads were washed with

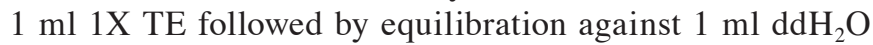

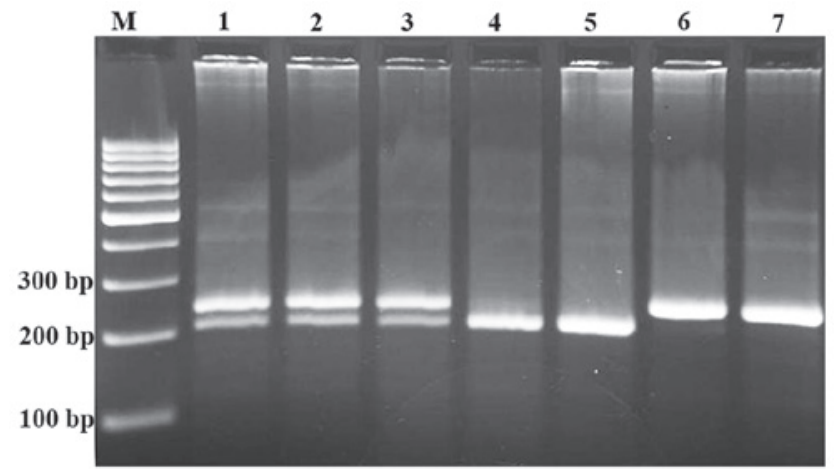

Figure 1. Representative polymerase chain reaction (PCR) products resolved by agarose gel electrophoresis to detect the presence or absence of the 32-bp deletion of the phosphatase and tensin homolog deleted on chromosome ten (PTEN) gene. M, DNA marker. Lanes 1-3, ins/del; lanes 4 and 5: del/del; lanes 6 and 7: ins/ins.

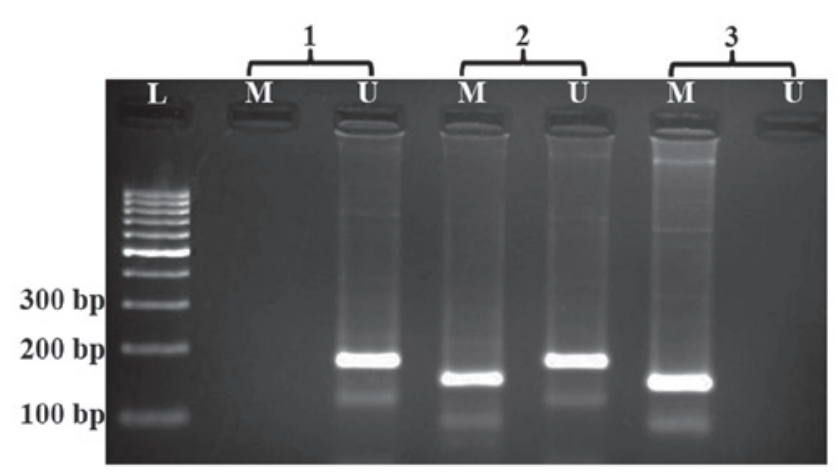

Figure 2. Electrophoresis pattern of promoter methylation of phosphatase and tensin homolog deleted on chromosome ten (PTEN) using methylationspecific PCR (MSP). L, DNA marker; M, methylated; U, unmethylated. Methylated primers amplify a 155 -bp product while unmethylated primers amplify 173-bp products. Sample 1, UU; sample 2, MU; sample 3, MM.

(1x15 min). The beads were used directly for PCRs. We have also kept beads at $-20^{\circ} \mathrm{C}$ for several weeks and never detected any loss of quality.

We developed a nested methylation-specific PCR (MSP) method for the detection of promoter methylation of PTEN with increased MSP sensitivity. First-stage PCR primers in the nested MSP recognize a bisulfite-treated template but do not discriminate between methylated and unmethylated alleles. In the second stage, two pairs of primers are used; one pair is specific for an unmethylated template and the other is specific for a methylated template. The forward and reverse primers for the first stage were 5'-TTTAGGGAGGGGGTTTGA-3' and 5'-CCATCCTCTTAATATCTCCT-3', respectively, producing a 529-bp amplicon that was used as a template for the second PCR stage once diluted 1:5. The second stage was performed as previously described (25). Primer sequences used to amplify an unmethylated product were 5'-TGGGTTTTGGAGGTT GTTGGT-3' (sense) and 5'-ACTTAACTCTAAACCACAA CCA-3' (antisense), which amplify a 173-bp product, and primer sequences for the methylated reaction were 5'-GGTTTCGGAGGTCGTCGGC-3' (sense) and 5'-CAACCG AATAATAACTACTACGACG-3' (antisense), generating a 155-bp product. 
Table I. Association of the 32-bp insertion/deletion polymorphism of the PTEN gene in individuals with and without MeS.

\begin{tabular}{|c|c|c|c|c|}
\hline Polymorphism & MeS (yes) & MeS (no) & OR $(95 \% \mathrm{CI})$ & P-value \\
\hline \multicolumn{5}{|c|}{ PTEN (32-bp ins/del) } \\
\hline Ins/ins & $44(29.1)$ & $37(24.8)$ & Ref. & - \\
\hline Ins/del & $70(46.4)$ & $72(48.3)$ & $0.81(0.47-1.41)$ & 0.471 \\
\hline Del/del & $37(24.5)$ & $40(26.8)$ & $0.77(0.41-1.45)$ & 0.431 \\
\hline Ins/del+del/del & $107(79)$ & $112(75.1)$ & $0.80(0.48-1.34)$ & 0.436 \\
\hline \multicolumn{5}{|l|}{ Alleles } \\
\hline Ins & $158(52)$ & $142(48)$ & Ref. & - \\
\hline Del & $144(48)$ & $152(52)$ & $0.84(0.61-1.16)$ & 0.288 \\
\hline
\end{tabular}

PTEN, phosphatase and tensin homolog deleted on chromosome ten; MeS (yes), patients with metabolic syndrome; MeS (no), patients without metabolic syndrome; OR, odds ratio; CI, confidence interval.

Table II. Frequency distribution of the PTEN promoter methylation in individuals with and without MeS.

\begin{tabular}{|c|c|c|c|c|}
\hline PTEN promoter methylation & MeS (yes) & MeS (no) & OR $(95 \% \mathrm{CI})$ & P-value \\
\hline UU & $129(85.4)$ & $112(75.2)$ & Ref. & - \\
\hline MU & $16(10.6)$ & $30(20.1)$ & $0.46(0.24-0.89)$ & 0.024 \\
\hline MM & $6(4.0)$ & $7(4.7)$ & $0.74(0.24-2.28)$ & 0.744 \\
\hline $\mathrm{MM}+\mathrm{MU}$ & $22(14.6)$ & $37(24.8)$ & $0.52(0.29-0.92)$ & 0.029 \\
\hline
\end{tabular}

PTEN, phosphatase and tensin homolog deleted on chromosome ten; MeS (yes), patients with metabolic syndrome; MeS (no), patients without metabolic syndrome; OR, odds ratio; CI, confidence interval; U, unmethylated; M, methylated.

The cycling conditions were as follows: $95^{\circ} \mathrm{C}$ for $10 \mathrm{~min}$, and 35 cycles of $95^{\circ} \mathrm{C}$ for $30 \mathrm{sec}, 57^{\circ} \mathrm{C}$ for $30 \mathrm{sec}$ and $72^{\circ} \mathrm{C}$ for $10 \mathrm{~min}$ with a final extension for $10 \mathrm{~min}$. The PCR products were verified on $2 \%$ agarose gel containing ethidium bromide and observed under UV light (Fig. 2).

Statistical analysis. The differences between the variables were assessed by the $\chi^{2}$ test or t-tests according to the data. The association between genotypes and MeS was assessed by computing the odds ratio (OR) and $95 \%$ confidence interval $(95 \% \mathrm{CI})$ from logistic regression analyses. $\mathrm{P}<0.05$ was considered to indicate a statistically significant difference. All statistical analyses were performed using SPSS 18 software.

\section{Results}

A total of 151 cases with MeS (50 male, 101 female; age, $41.98 \pm 14.65$ years) and 149 controls (45 male, 104 female; age, $43.53 \pm 15.96$ years) were enrolled in the study. There were no significant differences between the groups with regard to gender and age $(\mathrm{P}>0.05)$. As shown in Table I, no statistically significant difference was identified between the groups concerning 32-bp insertion/deletion polymorphism of the PTEN gene $\left(\chi^{2}=0.74\right.$, $\mathrm{P}=0.69)$. Our finding demonstrated that the 32-bp deletion polymorphism of PTEN is not a risk factor for MeS (Table I).

The promoter methylation status of the PTEN gene in the case and control groups is shown in Table II. The findings indicate that promoter methylation is a risk factor for $\mathrm{MeS}$ $(\mathrm{OR}=2.51,95 \% \mathrm{CI}=1.38-4.58, \mathrm{P}=0.002)$ in our population.

\section{Discussion}

In the present study, we analyzed the impact of genetic and epigenetic alterations of PTEN on the risk of developing $\mathrm{MeS}$ in a sample of an Iranian population. The PTEN 32-bp deletion demonstrated no effects on the predisposition to $\mathrm{MeS}$ since the frequency distribution of the del allele was similar in the MeS cases and the controls. However, PTEN promoter methylation was more prevalent in the individuals without MeS than the subjects with the syndrome. To the best of our knowledge, we report for the first time the correlation between promoter methylation of PTEN and decreased risk for MeS. Our findings suggest that hypermethylation of $\mathrm{CpG}$ sites in the promoter region of the PTEN gene is a possible mechanism accounting for downregulated or silenced PTEN expression.

$\mathrm{MeS}$ comprises a combination of risk factors for cardiovascular disease (CVD) and type 2 diabetes mellitus (T2DM). These factors include hyperglycemia, raised blood pressure, dyslipidemia primarily characterized by increased levels of triglycerides and low HDL-cholesterol and obesity (particularly with abdominal localization) (20). MeS is associated with a 2-fold increased risk for cardiovascular disease, 5-fold increased risk for T2DM and 1.5-fold increase 
in all-cause mortality (26). The prevalence of MeS varies worldwide and depends, in part, on lifestyle, gender, age and ethnicity $(20,27)$.

The expression and activity of the tumor suppressor PTEN are regulated by numerous and complex mechanisms, specifically in pathological conditions. These mechanisms include genetic alterations or epigenetic silencing of the gene by promoter methylation, which affect PTEN stability, localization, activity and interactions with other cellular partners (28). PTEN is mutated or deleted in $30 \%$ of all human cancers, and is thus, after p53, one of the most common tumor suppressors, the expression or activity of which is altered during carcinogenesis (29). A few studies have evaluated the 39-bp deletion variant located on intron 7 , close to the inton 7 -exon 8 splice site deletion of the PTEN gene. Ding et al (30) identified no association between the frequencies of this del variant in patients with HCC. By contrast, Zhou et al (31) suggested that this intronic deletion variant is not a mutation, but more likely a normal polymorphic variation, which has no functional effect on the expression of PTEN. In the present study we evaluated the 32-bp deletion of PTEN in intron 2 and identified no association with MeS.

In addition to germline mutations or deletion, the epigenetic silencing of PTEN through its promoter hypermethylation has been suggested as a potential mechanism contributing to PTEN downregulation. PTEN promoter methylation has been reported to be positively associated with increased risk for several types of cancer, including breast cancer $(32,33)$, cervical carcinoma (34) and thyroid tumors (35). However, no study has yet investigated this polymorphism in non-cancerous disease.

The liver-specific deletion of PTEN has been reported to correlate with insulin hypersensitivity, decreased serum leptin and body fat content and progression of fatty liver via insulin-induced fatty acid synthesis (36). Moreover, a number of studies indicate that PTEN is a major dysregulated cellular factor contributing to the development of a wide spectrum of disorders related to MeS, including T2DM, steatosis, steatohepatitis, fibrosis, cirrhosis, cardiac hypertrophy, heart failure, preconditioning and hypertension $(1,28,29,37)$.

In $\mathrm{MeS}$, in addition to an excess of circulating free fatty acids, the release of inflammatory cytokines or various adipokines, including leptin, resistin and adiponectin, have been reported to either up- or downregulate PTEN expression or activity in non-hepatic cells, thus leading to pathological conditions related to $\mathrm{MeS}$ (28).

PTEN is a tumor suppressor that regulates multiple cellular functions, including cell growth and survival, differentiation and proliferation, apoptosis, focal adhesion, invasion, migration and angiogenesis $(4,29)$. As a dual-specificity enzyme, PTEN is capable of dephosphorylating proteins and lipids. Through its phosphatase activity, PTEN downregulates the PI3K/Akt signaling pathway, while the protein phosphatase function of PTEN suppresses the integrin/FAK and Ras/MAPK/Erk1/2 pathways which are involved in the progression of cell cycle, migration and invasion $(5,8)$.

In conclusion, our findings demonstrate for the first time that the 32-bp deletion polymorphism of PTEN is nonfunctional in predisposing individuals to $\mathrm{MeS}$, but PTEN promoter hypermethylation protects against susceptibility to
MeS. Larger studies with individuals of different ethnicity are required to validate our findings.

\section{Acknowledgements}

This study was supported by a dissertation grant (MSc thesis of HR) from Zahedan University of Medical Sciences. The authors thank all subjects who willingly participated in the study.

\section{References}

1. Church T: Exercise in obesity, metabolic syndrome, and diabetes. Prog Cardiovasc Dis 53: 412-418, 2011.

2. Abete I, Goyenechea E, Zulet MA and Martinez JA: Obesity and metabolic syndrome: potential benefit from specific nutritional components. Nutr Metab Cardiovasc Dis 21 (Suppl 2): B1-B15, 2011.

3. Azimi-Nezhad M, Herbeth B, Siest G, et al: High prevalence of metabolic syndrome in Iran in comparison with France: what are the components that explain this? Metab Syndr Relat Disord 10: 181-188, 2012.

4. Waite KA and Eng C: Protean PTEN: form and function. Am J Hum Genet 70: 829-844, 2002

5. Weng L, Brown J and Eng C: PTEN induces apoptosis and cell cycle arrest through phosphoinositol-3-kinase/Akt-dependent and -independent pathways. Hum Mol Genet 10: 237-242, 2001.

6. Fernández S, García-García M and Torres-Alemán I: Modulation by insulin-like growth factor I of the phosphatase PTEN in astrocytes. Biochim Biophys Acta 1783: 803-812, 2008.

7. Maehama T and Dixon JE: The tumor suppressor, PTEN/MMAC1, dephosphorylates the lipid second messenger, phosphatidylinositol 3,4,5-trisphosphate. J Biol Chem 273: 13375-13378, 1998.

8. Leslie NR and Downes CP: PTEN: The down side of PI 3-kinase signalling. Cell Signal 14: 285-295, 2002.

9. Wijesekara N, Konrad D, Eweida M, et al: Muscle-specific PTEN deletion protects against insulin resistance and diabetes. Mol Cell Biol 25: 1135-1145, 2005.

10. Clément S, Krause U, Desmedt F, et al: The lipid phosphatase SHIP2 controls insulin sensitivity. Nature 409: 92-97, 2001

11. Qu Y, Sun L, Yang Z and Han R: Variation in the PTEN-induced putative kinase 1 gene associated with the increase risk of type 2 diabetes in northern Chinese. J Genet 90: 125-128, 2011.

12. Tsugawa K, Jones MK, Akahoshi T, et al: Abnormal PTEN expression in portal hypertensive gastric mucosa: a key to impaired PI 3-kinase/Akt activation and delayed injury healing? FASEB J 17: 2316-2318, 2003.

13. Hers I, Vincent EE and Tavaré JM: Akt signalling in health and disease. Cell Signal 23: 1515-1527, 2011.

14. Mocanu MM and Yellon DM: PTEN, the Achilles' heel of myocardial ischaemia/reperfusion injury? Br J Pharmacol 150: 833-838, 2007.

15. Butler M, McKay RA, Popoff IJ, et al: Specific inhibition of PTEN expression reverses hyperglycemia in diabetic mice. Diabetes 51: 1028-1034, 2002.

16. Besson A, Robbins SM and Yong VW: PTEN/MMAC1/TEP1 in signal transduction and tumorigenesis. Eur J Biochem 263: 605-611, 1999.

17. Keniry $\mathrm{M}$ and Parsons R: The role of PTEN signaling perturbations in cancer and in targeted therapy. Oncogene 27: 5477-5485, 2008.

18. Mirmohammadsadegh A, Marini A, Nambiar S, et al: Epigenetic silencing of the PTEN gene in melanoma. Cancer Res 66: 6546-6552, 2006.

19. Expert Panel on Detection, Evaluation, and Treatment of High Blood Cholesterol in Adults: Executive Summary of the Third Report of The National Cholesterol Education Program (NCEP) Expert Panel on Detection, Evaluation, and Treatment of High Blood Cholesterol in Adults (Adult Treatment Panel III). JAMA 285: 2486-2497, 2001

20. Kaykhaei MA, Hashemi M, Narouie B, et al: Prevalence of metabolic syndrome in adult population from Zahedan, southeast Iran. Iran J Public Health 41: 70-76, 2012.

21. Hashemi M, Kordi-Tamandani DM, Sharifi N, et al: Serum paraoxonase and arylesterase activities in metabolic syndrome in Zahedan, southeast Iran. Eur J Endocrinol 164: 219-222, 2011. 
22. Kordi-Tamandani DM, Hashemi M, Sharifi N, Kaykhaei MA and Torkamanzehi A: Association between paraoxonase-1 gene polymorphisms and risk of metabolic syndrome. Mol Biol Rep 39: 937-943, 2012.

23. Hashemi M, Moazeni-Roodi AK, Fazaeli A, et al: Lack of association between paraoxonase-1 Q192R polymorphism and rheumatoid arthritis in southeast Iran. Genet Mol Res 9: 333-339, 2010.

24. Tiwari SK, Manoj G, Prasanth K, et al: Simplified and versatile method for bisulfite-based DNA methylation analysis of small amounts of DNA. J Clin Lab Anal 23: 172-174, 2009.

25. Goel A, Arnold CN, Niedzwiecki D, et al: Frequent inactivation of PTEN by promoter hypermethylation in microsatellite instabilityhigh sporadic colorectal cancers. Cancer Res 64: 3014-3021, 2004

26. Mottillo S, Filion KB, Genest J, et al: The metabolic syndrome and cardiovascular risk a systematic review and meta-analysis. J Am Coll Cardiol 56: 1113-1132, 2010.

27. Cameron AJ, Shaw JE and Zimmet PZ: The metabolic syndrome: prevalence in worldwide populations. Endocrinol Metab Clin North Am 33: 351-375, 2004.

28. Peyrou M, Bourgoin L and Foti M: PTEN in non-alcoholic fatty liver disease/non-alcoholic steatohepatitis and cancer. Dig Dis 28: 236-246, 2010.

29. Vinciguerra M and Foti M: PTEN at the crossroad of metabolic diseases and cancer in the liver. Ann Hepatol 7: 192-199, 2008.

30. Ding J, Gao Y, Liu R, Xu F and Liu H: Association of PTEN polymorphisms with susceptibility to hepatocellular carcinoma in a Han Chinese population. DNA Cell Biol 30: 229-234, 2011.
31. Zhou XP, Hampel H, Roggenbuck J, Saba N, Prior TW and Eng C: A 39-bp deletion polymorphism in PTEN in African American individuals: implications for molecular diagnostic testing. J Mol Diagn 4: 114-117, 2002

32. Sadeq V, Isar $\mathrm{N}$ and Manoochehr T: Association of sporadic breast cancer with PTEN/MMAC1/TEP1 promoter hypermethylation. Med Oncol 28: 420-423, 2011.

33. Phuong NT, Kim SK, Lim SC, et al: Role of PTEN promoter methylation in tamoxifen-resistant breast cancer cells. Breast Cancer Res Treat 130: 73-83, 2011.

34. Rizvi MM, Alam MS, Ali A, Mehdi SJ, Batra S and Mandal AK: Aberrant promoter methylation and inactivation of PTEN gene in cervical carcinoma from Indian population. J Cancer Res Clin Oncol 137: 1255-1262, 2011.

35. Hou P, Ji M and Xing M: Association of PTEN gene methylation with genetic alterations in the phosphatidylinositol 3-kinase/AKT signaling pathway in thyroid tumors. Cancer 113: 2440-2447, 2008.

36. Rutledge AC and Adeli K: Fructose and the metabolic syndrome: pathophysiology and molecular mechanisms. Nutr Rev 65 S13-S23, 2007.

37. Ravi Y, Selvendiran K, Meduru S, et al: Dysregulation of PTEN in cardiopulmonary vascular remodeling induced by pulmonary hypertension. Cell Biochem Biophys: Dec 29, 2011 (Epub ahead of print). 